\title{
Correction to: Tree-Based Unrooted Phylogenetic Networks
}

\author{
A. Francis ${ }^{1}$ (D) K. T. Huber ${ }^{2}$ - V. Moulton ${ }^{2}$
}

Received: 24 October 2018 / Accepted: 30 October 2018 / Published online: 16 November 2018

(c) Society for Mathematical Biology 2018

\section{Correction to: Bull Math Biol (2018) 80:404-416 https://doi.org/10.1007/s11538-017-0381-3}

The level-5 example of a network presented in Fig. 4 of Francis et al. (2018) is treebased even though it states in the caption and in the text that this is not the case. This is because it contains the spanning tree with leaf-set $\{x, y\}$ given by the path $x, a, v_{1}, v_{2}, e, f, g, h, b, c, d, y$. The example should be replaced by the network in Fig. 1, which is level-5 and not tree-based. The network in Fig. 1 is not tree-based since any spanning tree with leaf-set $\{x, y\}$ must contain the edges $\{x, a\}$ and $\{d, y\}$ as well as one of the paths $b, h, h^{\prime}, c$ or $b, h^{\prime}, h, c$ and one of the paths $f, g, g^{\prime}, e$ or $f, g^{\prime}, g, e$. It follows that the spanning tree then must also contain the edges $\{a, b\}$, $\{c, d\},\{a, f\}$, and $\{e, d\}$, which is impossible. We thank Remie Janssen and Yukihiro Murakami for bringing this issue and replacement example to our attention.

Since drafting this erratum, other authors have independently pointed out the same error and made a more comprehensive corresponding fix (Fischer et al. 2018).

The original article can be found online at https://doi.org/10.1007/s11538-017-0381-3.

K. T. Huber

k.huber@uea.ac.uk

1 Centre for Research in Mathematics, Western Sydney University, Sydney, Australia

2 School of Computing Sciences, University of East Anglia, Norwich, UK 


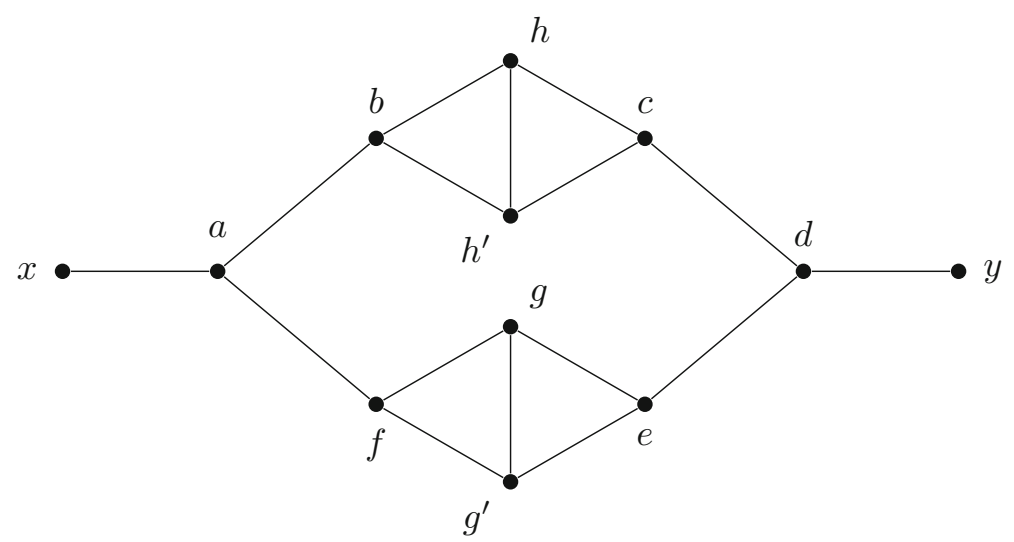

Fig. 1 A level-5 network that is not tree-based

\section{References}

Fischer M, Galla M, Herbst L, Long Y, Wicke K (2018) Non-binary treebased unrooted phylogenetic networks and their relations to binary and rooted ones. Preprint, arXiv:1810.06853v1, 16th Oct 2018

Francis A, Huber KT, Moulton V (2018) Tree-based unrooted phylogenetic networks. Bull Math Biol 80:404-416 